\title{
Breast Cancer or a Rear Diagnosis
}

\author{
Toktam Beheshtian ${ }^{1,}$, Faeze Yahyaei ${ }^{2}$, Shahin Ghassemi ${ }^{3}$ \\ ${ }^{1}$ Breast Diseases Department, Breast Cancer Research Center, Motamed Cancer \\ Institute, ACECR, Tehran, Iran \\ ${ }^{2}$ School of Medicine, Tehran University of Medical Sciences, Tehran, Iran \\ ${ }^{3}$ Department of Infectious Diseases, Iran University of Medical Sciences, Tehran, Iran \\ * Corresponding author: Toktam Beheshtian, Breast Diseases Department, Breast \\ Cancer Research Center, Motamed Cancer Institute, ACECR, Tehran, Iran. E-mail: \\ tbeheshtian@gmail.com
}

DOI: $10.21859 / \mathrm{mci}-01032$

Submitted: 24 March 2017

Revised: 20 May 2017

Accepted: 3 June 2017

ePublished: 11 June 2017

\section{Keywords:}

Tuberculosis

Breast Neoplasms

Mammography

Ultrasonography

\begin{abstract}
Introduction: Tuberculosis (TB) and its diagnosis are of great importance in the middleeast, according to the increasing burden of this disease and the large number of unreported cases. Less than $20 \%$ of all TB cases only have extra-pulmonary manifestations and breast TB is a rare form of extra-pulmonary TB. It includes about $1 \%$ of all breast lesions.

Case Presentation: This study presents an 83 year-old breast TB female case with the complaints of fatigue, lethargy, and fever. In the physical examination, the patient had a discharging abscess in upper outer quadrant of her right breast associated with edema and erythema, accompanied with multiple axillary and supraclavicular adenopathies. Imaging showed multiple cervical, axillary, and abdominal adenopathies. Clinical manifestations were highly suggestive of inflammatory breast carcinoma. Further investigations, including lymph node biopsy and Polymerase Chain Reaction (PCR) helped with the diagnosis of TB in the patient. The patient was treated with quadruple therapy of TB.

Conclusion: Because of its nonspecific clinical features, TB mastitis is often being misdiagnosed and underreported. Early diagnosis of breast TB could reduce patients' morbidity and also facilitate an effective treatment of patients with inflammatory breast lesions. TB should be the first differential diagnosis of granulomatous mastitis.
\end{abstract}

(C) 2017. Multidisciplinary Cancer Investigation

\section{INTRODUCTION}

Tuberculosis (TB) is of great importance in the middle east, as it is not yet under control [1]. Also, about $30 \%$ of TB cases are being underreported in the middle-east $[1,2]$. About $18 \%$ of tuberculosis cases only have extra-pulmonary manifestations. Breast TB is an extremely rare form of extra-pulmonary TB with prevalence of $1 \%$ of all breast lesions. It's prevalence is higher in endemic areas (4\%) [3]. TB mastitis most commonly presents itself with pain and as breast masses particularly in a woman [3]. Although it has been reported in men as well [4]. Thus, breast TB is a differential diagnosis for breast masses as well as breast carcinoma $[5,6]$.

\section{CASE PRESENTATION}

The case was an 83 year-old woman presented with fatigue, lethargy, fever, and axillary and supraclavicular adenopathies from several months ago. In physical examination, both breasts were edematous and erythematous and the skin was thickened in both breasts. There was an abscess in the upper outer quadrant of the right breast with a discharging sinus. In the past medical history, the patient had chronic kidney disease, ischemic heart disease, and hypertension, and she was under treatment with $50 \mathrm{mg}$ Losartan daily. Laboratory findings included hemoglobin $=9.5$, creatinine $=1.3$, erythrocyte sedimentation rate $(E S R=146)$, C-reactive protein $(\mathrm{CRP}=74)$, lactic acid dehydrogenase $(\mathrm{LDH}=533)$, and creatine phosphokinase $(\mathrm{CPK}=449)$, which increased to 860. Echocardiography and chest X-ray only revealed cardiomegaly. Sonography revealed multiple adenopathies with cystic changes in jugular and supraclavicular chains and multiple significant hypoechoic adenopathies in both axillas plus multiple hypoechoic masses with necrotic changes in upper outer quadrant of the right breast up to $4^{*} 3^{*} 2.5 \mathrm{~cm}$ due to abscess. In the mammography, there was bilateral skin thickening plus bilateral axillary adenopathies. Thoracoabdominal CT-scan showed infiltration in upper lobe of the left lung and paraaortic and internal and external iliac adenopathies. Spine and brain magnetic resonance imaging (MRI) were normal. Core-needle biopsy (CNB) from the abscess wall showed non-specific inflammatory reaction. The diagnosis was finally established by histopathology of axillary lymph node biopsy, which showed necrotizing lymphadenitis and acid fast stain- 
ing of the specimen was positive. Also, Polymerase Chain Reaction (PCR) was positive for TB bacilli. The patient was treated with quadruple therapy of TB with isoniazid, rifampin, pyrazinamide and ethambutol.

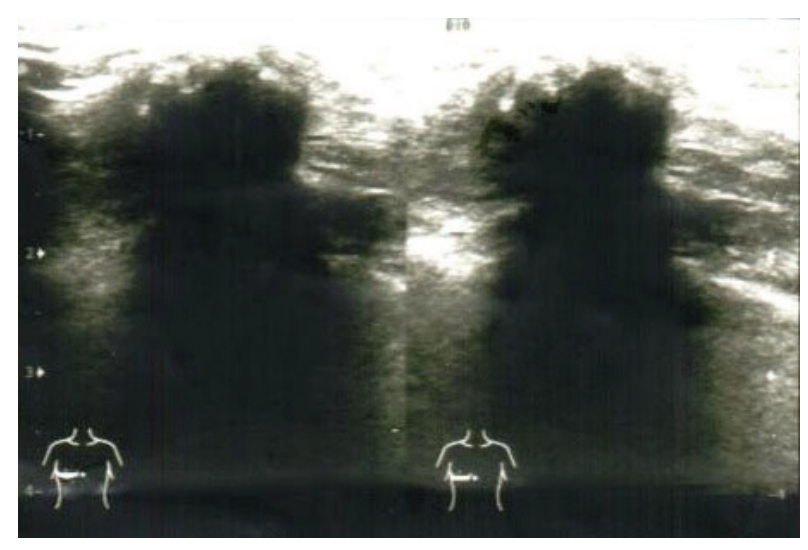

Figure 1: Hypoechoic Shadowing Irregular Border Mass

\section{DISCUSSION}

Clinical manifestations of breast TB are clinically and radiologically nonspecific, making it difficult to be diagnosed [7]. The main clinical features of TB mastitis include pain, lump, ulcer, abscess and less frequently Peau d'orange appearance and nipple purulent discharge. Lumps are usually solitary and located in the central or upper outer quadrant of the breast and abscesses could have discharging sinuses [8]. These clinical findings are similar to those of inflammatory breast carcinoma [5]. Inflammatory breast carcinoma distinctly presents itself with tenderness, warmth, and enlargement of involved breast; dermatologic manifestations include palpable masses, erythema, edema, induration, and Peau d'orange appearance [9]. TB mastitis is classified to 5 categories, including nodular tubercular mastitis, disseminated or confluent tubercular mastitis, sclerosing tubercular mastitis, tuberculous mastitis obliterans, and acute miliary tubercular mastitis [10]. About $35 \%$ of lesions in TB mastitis are compatible with malignancy in the physical examination; while about $45 \%$ of them have radiological features suggesting malignancy diagnosis [6]. Several methods are available to evaluate breast TB. Since patients with TB mastitis are usually being misdiagnosed and undergo numerous investigations, the most specific and sensitive modality to establish the diagnosis should be chosen.

Mantoux text is not useful as it is usually positive in adults, especially in endemic areas [8]. None of the radiological investigations, such as mammography, CT-scan and MRI, are useful in the diagnosis [8]. Mammography cannot differentiate between TB and carcinoma; yet it may help reduce the number of false positive cases of carcinoma, if the degree of density and trabecular thickening is evaluated carefully [11]. Mammogram in TB mastitis reveals lesions that are equal to their clinical size, unlike in breast carcinoma [12]. Disseminated variety of TB mastitis mimics inflammatory carcinoma and the radiographs show dense breast with thickened skin. These pictures lack the classic halo sign, which is found in fibroadenoma of the breast [13]. Disseminated variety mimics inflammatory carcinoma and the radiographs show dense breast with thickened skin [13]. Ultrasonography in tubercular mastitis mimics malignant tumors in $30 \%$ of cases [14]. Ultrasonography helps in differentiating cystic from solid lesions [18]. It reveals ill-defined hypoechoic masses in diffuse breast tuberculosis and increased echogenicity of the breast parenchyma is often with no definite mass in sclerosing breast tuberculosis [13, 15]. CT-scan is not very useful for diagnosis; yet it can show the extent of disease and the involvement of the lungs. Also, it can help with planning of the surgery and response to treatment assessment [8].

MRI of the breast reveals nonspecific features such as breast abscess. As well as CT-scan, MRI could be useful in determining extra-mammary involvement of the infection [8]. Furthermore, fine-needle aspiration cytology (FNAC) and $\mathrm{CNB}$ can be used for the diagnosis.

FNAC of inflammatory breast lesions is useful for diagnosis of TB mastitis. It's performance is higher when it is performed by a pathologist and is accompanied with further workups, such as microbiologic culture [16]. The FNACs sensitivity for breast tuberculosis is $73 \%$ when epithelioid cell granulomas and necrosis are seen [17], as a result FNAC may be inconclusive in a tubercular breast abscess and absence of acid fast bacilli cannot exclude TB mastitis [8]. Culture seems to be the gold standard for breast TB diagnosis; yet there are limitations, including the long duration required and frequent negative results [8]. PCR is highly sensitive, especially when the specimen is negative in culture. In the case of TB mastitis, PCR could help diagnose $50 \%$ of cases, which had been reported as granulomatous inflammation on cytology [18]. Thus, PCR is not absolute in diagnosing breast TB, because of the large number of false negative cases [19]. Histopathologic studies on specimen, which are obtained through open biopsy of breast lesions (lump, ulcer, sinus or from wall of the suspected tubercular breast abscess) almost always establishes the diagnosis of tuberculosis $[12,17]$. Histological feature of TB mastitis is granulomatous inflammation [8]. Differential diagnosis of granulomatous inflammation of breast are tuberculosis, sarcoidosis, Wegner's granulomatous, and idiopathic granulomatous mastitis [5].

Breast TB is mostly misdiagnosed due to its nonspecific manifestations and patients mostly undergo several investigations before the diagnosis is established. Since treatment of TB differs from other inflammatory lesions of the breast and also maltreatment may cause more morbidity than the disease itself, a high index of suspicion is required for timely diagnosis [8]. Furthermore, TB should be the first differential diagnosis of granulomatous mastitis if it is in an endemic area $[20]$ and/or when a breast abscess resists against adequate drainage and treatment with antibiotics, and if there is persistent discharging sinuses, the most appropriate modalities for diagnosing breast TB are histopathologic evaluation of abscess wall biopsy and culture [8]. 


\section{ACKNOWLEDGMENTS}

Not applicable.

\section{CONFLICT OF INTEREST}

The authors declare that they had no competing interests.

\section{ETHICS APPROVAL}

The ethics committee of Breast Cancer Research Center of ACECR approved the study.

\section{REFERENCES}

1. Al-Hajoj S, Varghese B. Tuberculosis in Saudi Arabia: the journey across time. J Infect Dev Ctries. 2015;9(3):222-31. DOI: 10.3855/jidc.5296 PMID: 25771458

2. Huseynova S, Hashim DS, Tbena MR, Harris R, Bassili A, Abubakar I, et al. Estimating tuberculosis burden and reporting in resource-limited countries: a capture-recapture study in Iraq. Int J Tuberc Lung Dis. 2013;17(4):462-7. DOI: 10.5588/ijtld.12.0209 PMID: 23485379

3. Marinopoulos S, Lourantou D, Gatzionis T, Dimitrakakis C, Papaspyrou I, Antsaklis A. Breast tuberculosis: Diagnosis, management and treatment. Int J Surg Case Rep. 2012;3(11):548-50. DOI: 10.1016/j.jijscr.2012.07.003 PMID: 22918083

4. Cantisani C, Lazic T, Salvi M, Richetta AG, Frascani F, De Gado F, et al. Male tuberculous mastitis: a rare entity. Clin Ter. 2013;164(4):e293-5. DOI: 10.7417/CT.2013.1591 PMID: 24045526

5. Brouwer A, Degrieck N, Rasschaert M, Lockefeer F, Huizing M, Tjalma W. Tuberculous mastitis presenting as a lump: a mimicking disease in a pregnant woman case report and review of literature. Acta Clin Belg. 2014;69(5):389-94. DOI: 10.1179/2295333714Y.0000000048 PMID: 25056489

6. Kilic MO, Saglam C, Agca FD, Terzioglu SG. Clinical, diagnostic and therapeutic management of patients with breast tuberculosis: Analysis of 46 Cases. Kaohsiung J Med Sci. 2016;32(1):27-31. DOI: 10.1016/j.kjms.2015.12.005 PMID: 26853172
7. Kumar M, Chand G, Nag VL, Maurya AK, Rao RN, Agarwal S, et al. Breast tuberculosis in immunocompetent patients at tertiary care center: A case series. Journal of Research in Medical Sciences : The Official Journal of Isfahan University of Medical Sciences. 2012;17(2):199-202. PMID: PMC3525043

8. Tewari M, Shukla HS. Breast tuberculosis: diagnosis, clinical features \& management. Indian J Med Res. 2005;122(2):10310. PMID: 16177466

9. Bussolati G, Badve S. WHO Classification of Tumours of the Breast: World Health Organization; 2012.

10. Vassilakos P. Tuberculosis of the breast: cytologic findings with fine-needle aspiration. A case clinically and radiologically mimicking carcinoma. Acta Cytol. 1973;17(2):160-5. PMID: 4511405

11. Al-Marri MR, Aref E, Omar AJ. Mammographic features of isolated tuberculous mastitis. Saudi Med J. 2005;26(4):64650. PMID: 15900377

12. Shinde SR, Chandawarkar RY, Deshmukh SP. Tuberculosis of the breast masquerading as carcinoma: a study of 100 patients. World J Surg. 1995;19(3):379-81. PMID: 7638992

13. Popli M. Pictorial essay :Tuberculosis of the breast. Indian J Radiol Image. 1999;9(3):127-32.

14. Sakr AA, Fawzy RK, Fadaly G, Baky MA. Mammographic and sonographic features of tuberculous mastitis. Eur J Radiol. 2004;51(1):54-60. DOI: 10.1016/S0720-048X(03)00230-4 PMID: 15186885

15. Oh KK, Kim JH, Kook SH. Imaging of tuberculous disease involving breast. Eur Radiol. 1998;8(8):1475-80. DOI: 10.1007/s003300050578 PMID: 9853240

16. Nemenqani D, Yaqoob N. Fine needle aspiration cytology of inflammatory breast lesions. J Pak Med Assoc. 2009;59(3):167-70. PMID: 19288945

17. Kakkar S, Kapila K, Singh MK, Verma K. Tuberculosis of the breast. A cytomorphologic study. Acta Cytol. 2000;44(3):292-6. PMID: 10833980

18. Nalini G, Kusum S, Barwad A, Gurpreet S, Arvind R. Role of polymerase chain reaction in breast tuberculosis. Breast Dis. 2015;35(2):129-32. DOI: 10.3233/BD-140390 PMID: 25267370

19. Katoch VM. Newer diagnostic techniques for tuberculosis Indian J Med Res. 2004;120(4):418-28. PMID: 15520490

20. Mirsaeidi SM, Masjedi MR, Mansouri SD, Velayati AA. Tuberculosis of the breast: report of 4 clinical cases and literature review. East Mediterr Health J. 2007;13(3):670-6. PMID: 17687841 